\title{
Early Functional Connectivity Predicts Recovery from Visual Field Defects after Stroke
}

\author{
Yong-Hwan Kim, , ${ }^{\mathrm{a}}$ A-Hyun Cho, ${ }^{\mathrm{b}, *}$ Dongho Kim, ${ }^{\mathrm{a}}$ Seung Min Kim, ${ }^{\mathrm{c}}$ Hyun Taek Lim, ${ }^{\mathrm{d}}$ Sun U. Kwon, ${ }^{\mathrm{e}}$ \\ Jong S. Kim, ${ }^{\mathrm{e}}$ Dong-Wha Kang, \\ ${ }^{a}$ Asan Institute for Life Sciences, Asan Medical Center, Seoul, Korea \\ ${ }^{b}$ Department of Neurology, Yeouido St. Mary's Hospital, College of Medicine, The Catholic University of Korea, Seoul, Korea \\ 'Department of Neurology, Veterans Health Service Medical Center, Seoul, Korea \\ ${ }^{d}$ Department of Ophthalmology, Asan Medical Center, University of Ulsan College of Medicine, Seoul, Korea \\ eDepartment of Neurology, Asan Medical Center, University of Ulsan College of Medicine, Seoul, Korea
}

Background and Purpose We aimed to assess whether early resting-state functional connectivity (RSFC) changes measured via functional magnetic resonance imaging (fMRI) could predict recovery from visual field defect (VFD) in acute stroke patients.

Methods Patients with VFD due to acute ischemic stroke in the visual cortex and age-matched healthy controls were prospectively enrolled. Serial resting-state (RS)-fMRI and Humphrey visual field (VF) tests were performed within 1 week and at 1 and 3 months (additional VF test at 6 months) after stroke onset in the patient group. The control group also underwent RS-fMRI and a Humphrey VF test. The changes in RSFCs and VF scores (VFSs) over time and their correlations were investigated.

Results In 32 patients (65 \pm 10 years, 25 men), the VFSs were lower and the interhemispheric RSFC in the visual cortices was decreased compared to the control group ( $n=15,62 \pm 6$ years, seven men). The VFSs and interhemispheric RSFC in the visual cortex increased mainly within the first month after stroke onset. The interhemispheric RSFC and VFSs were positively correlated at 1 month after stroke onset. Moreover, the interhemispheric RSFCs in the visual cortex within 1 week were positively correlated with the follow-up VFSs.

Conclusions Interhemispheric RSFCs in the visual cortices within 1 week after stroke onset may be a useful biomarker to predict long-term VFD recovery.

Keywords Infarction, posterior cerebral artery; Visual fields; Recovery of function; Magnetic resonance imaging

\author{
Correspondence: Dong-Wha Kang \\ Department of Neurology, Asan \\ Medical Center, University of Ulsan \\ College of Medicine, 88 Olympic-ro \\ 43-gil, Songpa-gu, Seoul 05505, Korea \\ Tel: $+82-2-3010-3440$ \\ Fax: +82-2-474-4691 \\ E-mail:dwkang@amc.seoul.kr
}

Received: October 22, 2018 Revised: February 27, 2019 Accepted: March 3, 2019

*These authors contributed equally to the manuscript as first author.

\section{Introduction}

Visual field defect (VFD) is a main disability after posterior circulation stroke. ${ }^{1}$ Permanent VFDs impair quality of life. Furthermore, rehabilitation methods for VFD improvement remain very limited. ${ }^{2-5}$ Unraveling the mechanism of VFD recovery after stroke would help us identify the optimal methods for ad- vanced rehabilitation.

Functional recovery after stroke is associated with neural plasticity. Contralesional shift in activation and evolution to later ipsilesional activation, recruitment of additional regions, and activation of ipsilesional surviving regions occur during stroke recovery. ${ }^{6-9}$ There is a growing interest in intrinsic neuronal networks measured by functional magnetic resonance im- 
aging (fMRI), which shows resting-state functional connectivity (RSFC) between distinct regions based on low-frequency spontaneous fluctuations in the human brain..$^{10-13}$ In comparison with task-based fMRI, analysis in the steady state is less susceptible to confounding by unrelated brain regions. Restingstate (RS)-fMRI is a reliable method for evaluating the functional connectivity of distant brain regions, ${ }^{13,14}$ it has many useful clinical applications and is used to elucidate changes in functional connectivity in various brain disorders. ${ }^{15-18}$

However, the neural mechanism of RSFC associated with VFD recovery after stroke remains unclear. Herein, we aimed to assess whether RSFC would spontaneously change after acute stroke and whether early RSFC could predict long-term VFD recovery.

\section{Methods}

\section{Subjects and study design}

We prospectively enrolled acute ischemic stroke patients within 1 week of symptom onset between September 2011 and December 2014. We included patients who were aged $>20$ years, had VFDs such as homonymous hemianopia or quadrantanopia, had acute ischemic stroke in a unilateral posterior cerebral arterial territory confirmed by diffusion-weighted imaging (DWI) MRI, had a first-ever stroke, and did not receive thrombolytic therapy. Patients who newly developed neurologic events during the follow-up period (i.e., recurrent stroke or hemorrhagic transformation) were excluded from the final analysis. Clinical variables including demographic data and stroke risk factors were obtained. The onset time was defined as that when the patients were last known to have no visual disturbance. Agematched healthy controls were also prospectively enrolled.

Serial RS-fMRI and Humphrey visual field (VF) tests were performed within 1 week and at 1 and 3 months (additional VF test at 6 months) after stroke onset in the patient group. The age-matched healthy control group also underwent RS-fMRI and a Humphrey VF test. This study was approved by the Institutional Review Board of Asan Medical Center. Written informed consent was obtained from the subjects or their legal representatives.

\section{Assessment of VFDs}

VFs were quantitatively measured with a Humphrey field analyzer (HFA 750i, Zeiss-Humphrey, Leandro, CA, USA). The HFA tests the VFs of each eye using the light detection task. The VF for each eye was tested using the central 30-2 threshold SITAFast protocol. The HFA provides the total deviation scores in decibels $(\mathrm{dB})$, which represent the difference in light detection performance between the test results and age-matched normal values at each tested retinal point. In this study, the total deviation scores were averaged across eyes, and the mean total deviation (MTD) score was then calculated by averaging the scores in both the affected and unaffected hemifields (e.g., the affected hemifield is the left hemifield for left quadrantanopia or hemianopia patients). A single VF score (VFS) was generated by subtracting the MTDs in the unaffected hemifield from the affected hemifield for statistical analysis.

\section{MRI acquisition}

DWI was performed to diagnose acute ischemic stroke. The ischemic lesion mask was extracted in the native DWI space using the FSL View toolbox in FMRIB (Oxford Center for Functional Magnetic Resonance Imaging of the Brain) Software Library (FSL). ${ }^{19}$ The ischemic lesion was measured via manual delineation of the DWI lesion by an investigator (Y.H.K.) under the supervision of a stroke neurologist (D.W.K.) blinded to all clinical information. A b0 image was used for transformation from the DWI space to a high-resolution T1-weighted image via FMRIB's Linear Image Registration Tool with the normalized mutual information cost function. The lesion mask in the diffusion space was transformed into the standard Montreal Neurological Institute template to show the infarct distribution of VFD patients (Supplementary Figure 1).

RS-fMRI scans and high-resolution T1-weighted images were acquired using a 3.0-T Philips (Philips Achieva, Philips Medical Systems, Amsterdam, the Netherlands) MRI scanner. Patients were instructed to keep their eyes closed and to minimize the performance of distinct cognitive tasks during the fMRI sessions. Thirty-one contiguous slices without a gap aligned to the anterior commissure-posterior commissure line were acquired using a gradient-echo echo-planar imaging pulse sequence (repetition time/echo time, 2,000/30 ms; flip angle, $90^{\circ}$; voxel size, $1.5625 \times 1.5625 \times 3.5 \mathrm{~mm}^{3}$; field-of-view, $200 \times 200 \mathrm{~mm}^{2}$; 120 volumes). High-resolution T1-weighted magnetic resonance (MR) images were acquired with the magnetization-prepared rapid acquisition with gradient echo (MPRAGE) sequence (repetition time/echo time, 9.9/4.6 ms; flip angle, $8^{\circ}$; voxel size, $1 \times 1 \times 1 \mathrm{~mm}^{3}$ ).

\section{fMRI data preprocessing}

The first five volumes of fMRI data were discarded to achieve signal equilibrium (i.e., magnetization stability) and participant adaptation to scanning noise, and then 115 volumes were used for the RSFC analysis. RS-fMRI scans were preprocessed using FSL that included motion correction to the first fMRI scan and brain extraction. The relative root-mean-squared frame-wise 
displacement (FD-RMS) metric was used to assess head motion during fMRI scanning. ${ }^{20}$ We excluded subjects with marked head motion, including those who had either $>0.3 \mathrm{~mm}$ of average FD-RMS or $>10 \%$ of volumes (i.e., $>11$ of 115 volumes) with rapid motion (i.e., $>0.5 \mathrm{~mm}$ ) at any follow-up time.

Data were passed through several additional preprocessing steps: (1) tissue-based regressors were computed based on FreeSurfer segmentation for cerebrospinal fluid (CSF) and white matter (WM) masks, which were eroded using a 7-mm fullwidth-at-half-maximum Gaussian kernel to avoid a partial volume effect: ${ }_{1}^{21}$ (2) removal by regression of the following sources of spurious variance: (a) average signal within the brain area, (b) 24-head motion parameters from the motion correction, (c) minimal number of principal components that explained at least $50 \%$ of the variance from the merged mask including the WM, CSF, and edge of brain (i.e., aCompCor50), ${ }_{1}^{22}$ (d) scrubbing volumes based on FD-RMS $>0.5 \mathrm{~mm}_{1}^{.23}$ and (3) temporal filtering retaining frequencies in the 0.01 to $0.1-\mathrm{Hz}$ band.

\section{RSFC analysis}

Automatically-parcellated gray matter regions provided by FreeSurfer were used to define the regions of interest (ROIs) (Supplementary Figure 2). ${ }^{24}$ To generate pair-wise connectivity matrices, the time courses of all voxels within a ROI were averaged. RSFC was then computed between each ROI timeseries using Fisher z-transformed Pearson correlation. A total of 3,321 RSFCs from the 82 ROls were calculated.

\section{Statistical analysis}

In the VFD patient group, one-way repeated-measures analysis of variance (ANOVA) and paired t-test were used to assess the presence of alterations across time points in terms of the VFS (i.e., 1 week and 1, 3, and 6 months) and RSFC (i.e., 1 week and 1 and 3 months), respectively. Post hoc paired t-tests were performed between adjacent time points. Unpaired t-tests were performed to assess the differences in RSFC and the VFS between age-matched healthy controls and VFD patients. Correlation analysis was performed between RSFC and the VFS to assess neurobehavioral correlations in VFD patients. For the VFS analysis, Bonferroni correction was applied for multiple comparisons (i.e., number of comparisons $=6$ for post hoc paired $t$ tests between time points in the patient group and 4 for unpaired t-tests between the control and patient groups). The significance level was determined at $\alpha<0.05$. Because of the markedly large number of RSFCs, the network-based statistics (NBS) procedure was applied with 5,000 permutations (RSFC significance level at t-score $>3.0$ and network-extent $\alpha<0.05) .{ }^{25}$

\section{Results}

\section{Subjects}

During the study period, 52 patients admitted to our stroke center with VFDs caused by posterior cerebral artery infarction were screened. Of these, 20 patients were excluded (nine refused to provide consent, seven had a hemorrhagic transformation, two had a recurrent stroke during the follow-up period, and two had large head motion in RS-fMRI acquisition). Finally, 32 VFD patients were included in this study. In addition, 15 age-matched healthy control subjects were enrolled. Healthy control subjects underwent a Humphrey VF test with perimetry and one MRI session. Demographics, clinical features, and time points of perimetry and MRI scans of the subjects are presented in Table 1.

In the present study, the infarct location was matched across VFD patients to a location on the right hemisphere by inverting the MRI scans along the $x$-axis for those who had infarction in the left hemisphere ( $n=16$ of 32 patients). Subsequently, the

Table 1. Characteristics of the subjects

\begin{tabular}{lcccc}
\hline & $\begin{array}{c}\text { VFD patients } \\
(n=32)\end{array}$ & $\begin{array}{c}\text { Healthy } \\
\text { controls } \\
(n=15)\end{array}$ & \multicolumn{2}{c}{ Group differences } \\
\cline { 5 - 6 } Variable & $64.8 \pm 10.4$ & $62.0 \pm 5.5$ & 0.33 & $\begin{array}{c}\text { t score or } \\
X^{2}\end{array}$ \\
\hline Age (yr) & $25(78.1)$ & $7(46.7)$ & 0.03 & 4.65 \\
Male sex & $16(50.0)$ & - & - & - \\
Infarct in the left hemi- \\
sphere
\end{tabular}

Values are presented as mean \pm standard deviation or number (\%). A two-sample t-test $(\mathrm{t})$ or chi-square $\mathrm{t}$-test $\left(\mathrm{X}^{2}\right)$ was used for statistical analysis. VFD, visual field defect. 
hemisphere concept was transformed from right and left to the ipsilesional and contralesional hemispheres. Considering the asymmetry of brain function and anatomy, MRI analyses were performed by inverting across the $\mathrm{x}$-axis in the healthy controls ( $n=7$ of 15 controls).

\section{Recovery of VFDs}

The recovery of MTD in the affected and unaffected hemifields was evaluated (Figure 1A). In the study patients ( $n=32)$, compared to healthy controls $(n=15$; mean \pm standard error of MTD, $-0.9 \pm 0.5 \mathrm{~dB}$ ), VFDs were observed in both the affected (uncorrected; $P<0.001$ for all the time points; $-24.1 \pm 1.4 \mathrm{~dB}$ at 1 week; $-19.6 \pm 1.7 \mathrm{~dB}$ at 1 month; $-18.3 \pm 1.8 \mathrm{~dB}$ at 3 months; and $-18.6 \pm 1.7 \mathrm{~dB}$ at 6 months) and unaffected hemifields (uncorrected; $P<0.001$ for all the time points; $-5.2 \pm 0.8 \mathrm{~dB}$ at 1 week; $-4.5 \pm 0.8 \mathrm{~dB}$ at 1 month; $-4.3 \pm 0.7 \mathrm{~dB}$ at 3 months; and $4.1 \pm 0.7 \mathrm{~dB}$ at 6 months).

There was a significant change in MTD in the affected hemifield (uncorrected; $P<0.001, F[3,93]=25.35$ ), but no significant change in the unaffected hemifield (uncorrected; $P=0.058$; $\mathrm{F}[3,93]=2.59)$ over time. Improved MTD in the affected hemifield was observed within 1 month (MTD change, $4.4 \pm 1.0 \mathrm{~dB}$; Bonferroni corrected $P<0.001, t[31]=4.7$ from 1 week) and at 3 months after stroke (MTD change, $1.3 \pm 0.4 \mathrm{~dB}$; Bonferroni corrected $P=0.016, \mathrm{t}[31]=2.7$ from 1 month). There was no significant improvement after 3 months of stroke onset (MTD change, $-0.3 \pm 0.4 \mathrm{~dB}$ from 3 to 6 months; uncorrected $P=0.53$, $\mathrm{t}[31]=-0.6)$.

The MTDs in the unaffected hemifield did not show significant improvement during the follow-up period (MTD change
$[0.8 \pm 0.4 \mathrm{~dB}$ from 1 week to 1 month; uncorrected $P=0.075$, $\mathrm{t}(31)=1.8] ;[0.2 \pm 0.4 \mathrm{~dB}$ from 1 month to 3 months, uncorrected $P=0.65, \mathrm{t}(31)=0.46] ;$ and $[0.2 \pm 0.3 \mathrm{~dB}$ from 3 months to 6 months; uncorrected $P=0.52, \mathrm{t}(31)=0.65]$ ).

For the VFS, which represents a single value for a time point in a subject by subtracting the unaffected MTD from the affected MTD, compared to healthy controls $(n=15$, mean \pm standard error of VFS, $-0.1 \pm 0.2 d B)$, patients with VFDs $(n=32)$ had lower VFSs within 1 week (uncorrected $P<0.001 ;$ VFS, $-18.8 \pm 1.2 \mathrm{~dB}$ ), at 1 month (uncorrected $P<0.001 ; V F S,-15.2 \pm 1.4 \mathrm{~dB}$ ), at 3 months (uncorrected $P<0.001 ;-14.0 \pm 1.5 \mathrm{~dB}$ ) and at 6 months (uncorrected $P<0.001 ;-14.5 \pm 1.5 \mathrm{~dB}$ ) after stroke onset (Figure 1B).

On the one-way repeated-measures ANOVA across time, there was a significant change in the VFS (uncorrected $P<0.001, F[3,93]=13.13)$. Improvement in VFS was observed within 1 month (VFS change, $3.7 \pm 1.1 \mathrm{~dB}$; Bonferroni corrected $P=0.013, t[31]=3.4$ from 1 week). There was no significant increase after 1 month of stroke onset (IVFS change $1.1 \pm 0.5 \mathrm{~dB}$ from 1 month to 3 months; Bonferroni corrected $P=0.13$, $\mathrm{t}(31)=2.43$ ]; and [VFS change, $-0.5 \pm 0.4 \mathrm{~dB}$ from 3 months to 6 months; uncorrected $P=0.2, \mathrm{t}(31)=-1.3]$ ).

\section{Alterations in RSFC after stroke}

Compared to healthy controls, VFD patients showed lower levels of interhemispheric RSFCs in the occipital lobe within 1 week (NBS corrected $P=0.042$ ) (Figure 2A). The ROls with lower levels of interhemispheric RSFCs in the occipital lobes, compared to those of the healthy controls, were the bilateral cuneus, pericalcarine, and lingual cortices. However, there were no significant differences in RSFC between the control and patient
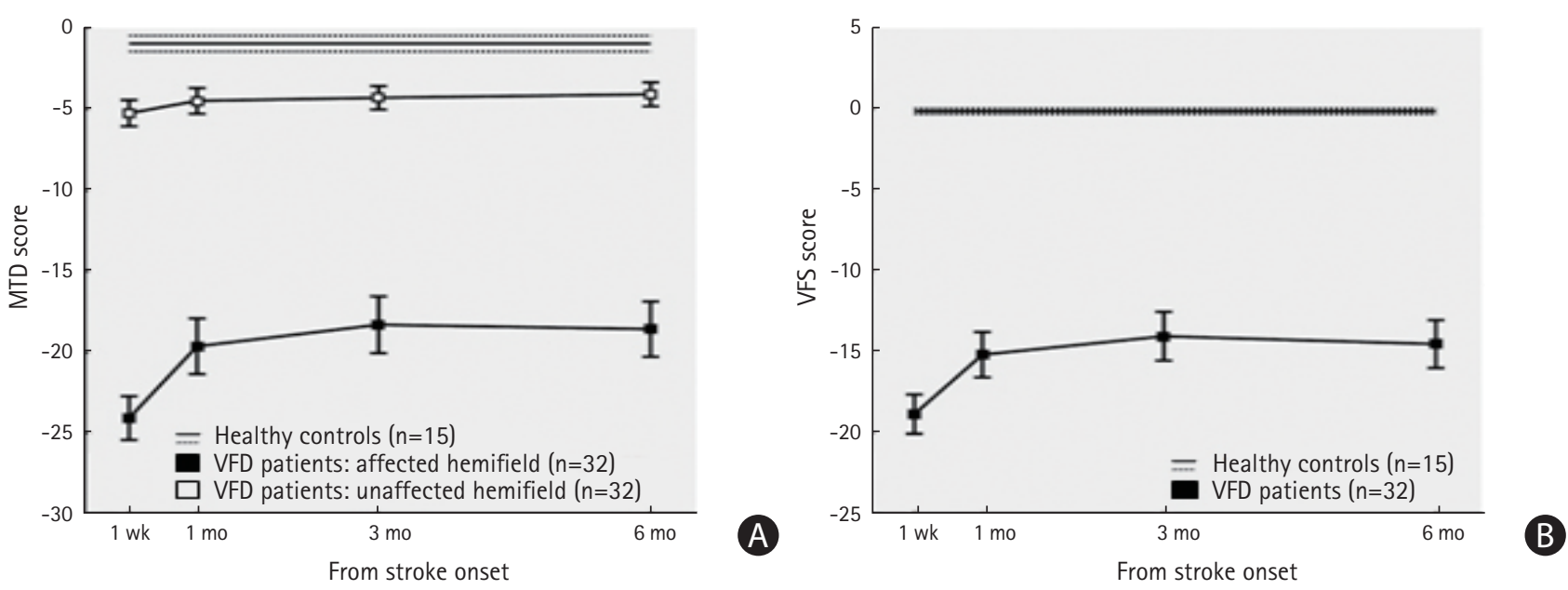

Figure 1. Temporal changes in the visual field score (VFS) after stroke. (A) Bar plot of mean total deviation (MTD) scores in the affected and unaffected hemifields over time are shown. (B) Bar plot of VFS changes over time are shown. VFSs were calculated in each subject by subtracting the MTDs in the unaffected hemifield from the affected hemifield. Markers with squares indicate the means and standard errors of visual field defect (VFD) patients ( $n=32$ ). Horizontal solid and dotted lines indicate the means and standard errors of age-matched healthy subjects $(n=15)$. 
groups at 1 and 3 months (Supplementary Figure 3 for qualitative RSFC level changes in VFD patients compared to healthy controls).

On the one-way repeated-measures ANOVA across time, there were no significant network changes (NBS corrected $P>0.05)$. However, from the paired t-test between 1 week and 1 month, increased RSFCs were observed in broad regions, such as the rostral/caudal anterior cingulate cortices, superior fron- tal gyri, Heschl's gyrus, middle/inferior temporal gyri, bank of the superior temporal sulcus, and amygdala (NBS corrected $P=0.028$ ) (Figure 2B). At 3 months after stroke, decreased RSFCs compared to within 1 week were observed in the contralesional regions of the cuneus, pericalcarine, lingual, lateral occipital, fusiform, parahippocampal, and superior/transverse temporal gyri and the ipsilesional thalamus (NBS corrected $P=0.006$ ) (Figure $2 \mathrm{C}$ ).
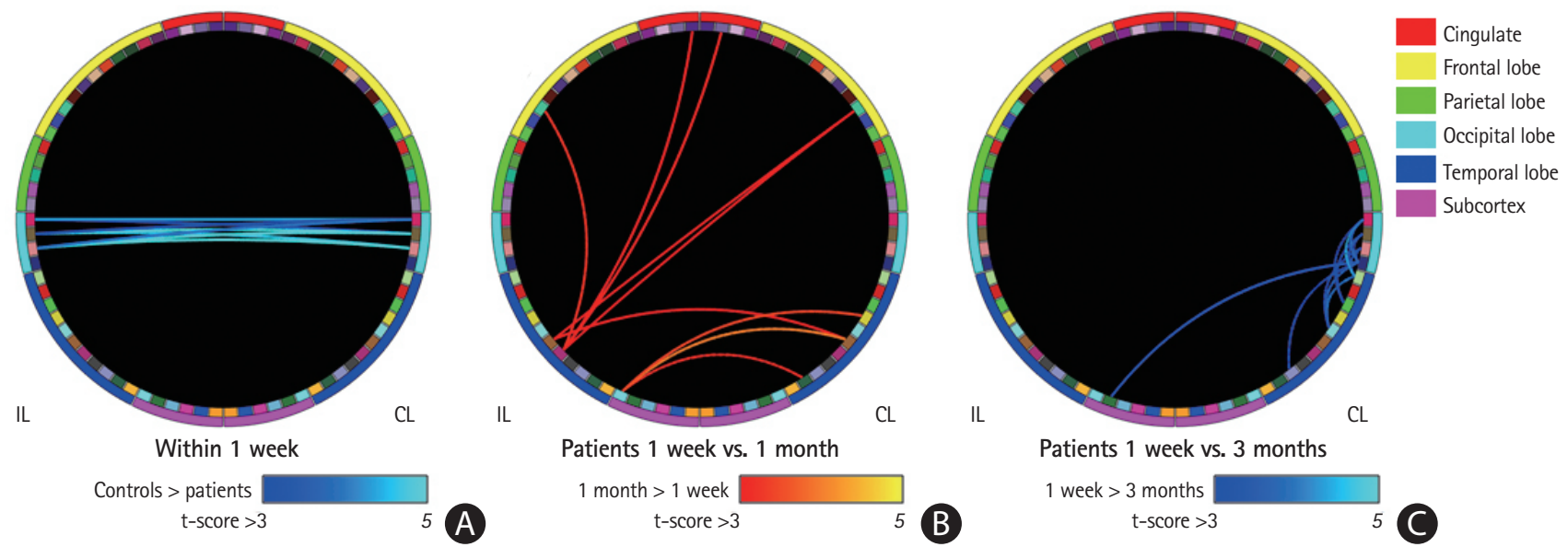

Figure 2. Resting-state functional connectivity (RSFC) changes after stroke. Circular representation of significance levels from statistical tests on RSFC for each paired region of interest (ROI; please see Supplementary Figure 2). (A) Results from two-sample t-test between visual field defect (VFD) patients ( $n=32$ ) within 1 week from stroke onset and age-matched healthy controls $(H C ; n=15)$. Compared to HC, VFD patients within 1 week after stroke onset showed lower levels of interhemispheric RSFCs in the occipital lobe (blue to light blue colors for significance levels: lighter colors indicate higher negative correlation). (B) Results from paired t-test between 1 week and 1 month in VFD patients. Significance levels of increased RSFCs between within 1 week and 1 month after stroke are shown with red to yellow colors in the Circos plot (yellow colors indicate higher positive correlation). (C) Results from paired t-test between 1 week and 3 months in VFD patients. Significance levels of decreased RSFCs between within 1 week and 3 months after stroke are shown with blue to light blue colors in the Circos plot. IL, ipsilesional hemisphere (i.e., left half of the Circos plot); $\mathrm{CL}$, contralesional hemisphere (i.e., right half of the Circos plot).

IL

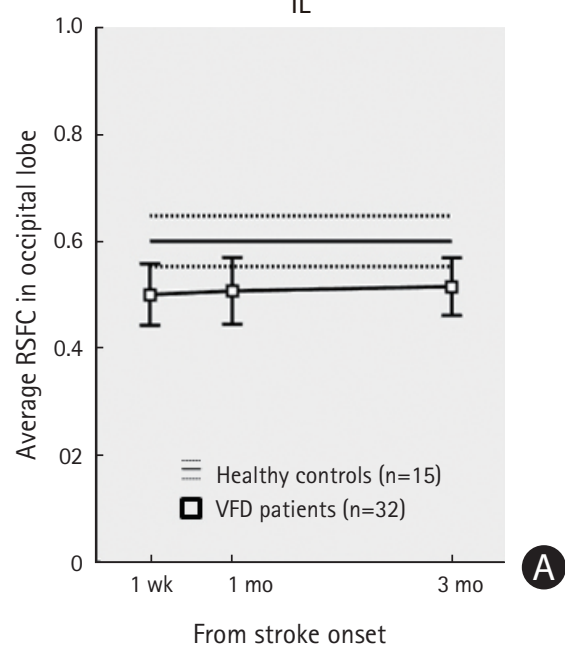

Between hemispheres

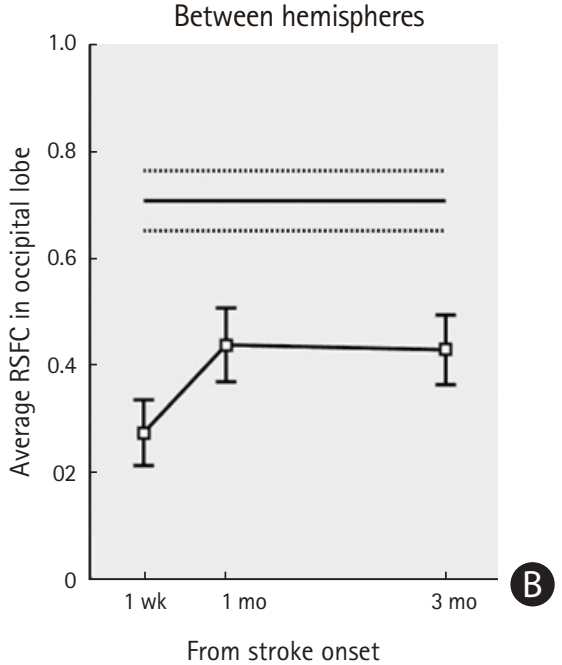

$\mathrm{CL}$

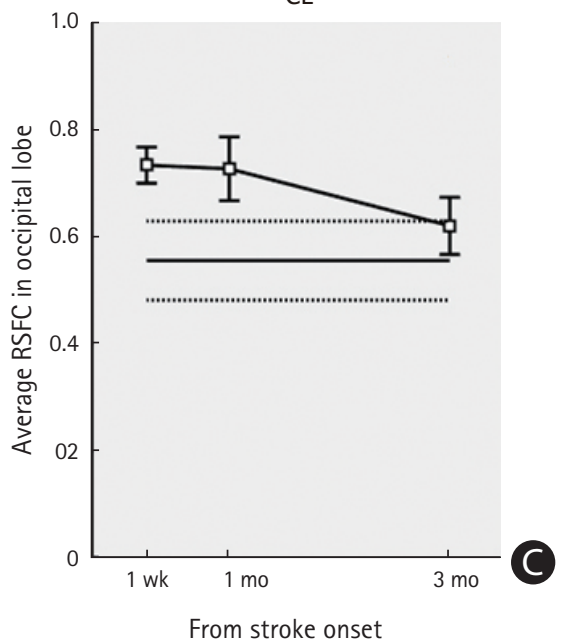

Figure 3. Resting-state functional connectivity (RSFC) changes in the occipital lobe. Bar plots of the average RSFC in the occipital lobe including the cuneus, pericalcarine, lingual, and lateral occipital cortex region of interest. Average RSFCs of within the (A) ipsilesional hemisphere (IL), (B) between hemispheres (middle), and (C) within the contralesional hemisphere (CL) are shown. Markers with squares indicate the means and standard errors of visual field defect patients $(n=32)$. Horizontal solid and dotted lines indicate the means and standard errors of age-matched healthy subjects $(n=15)$. 


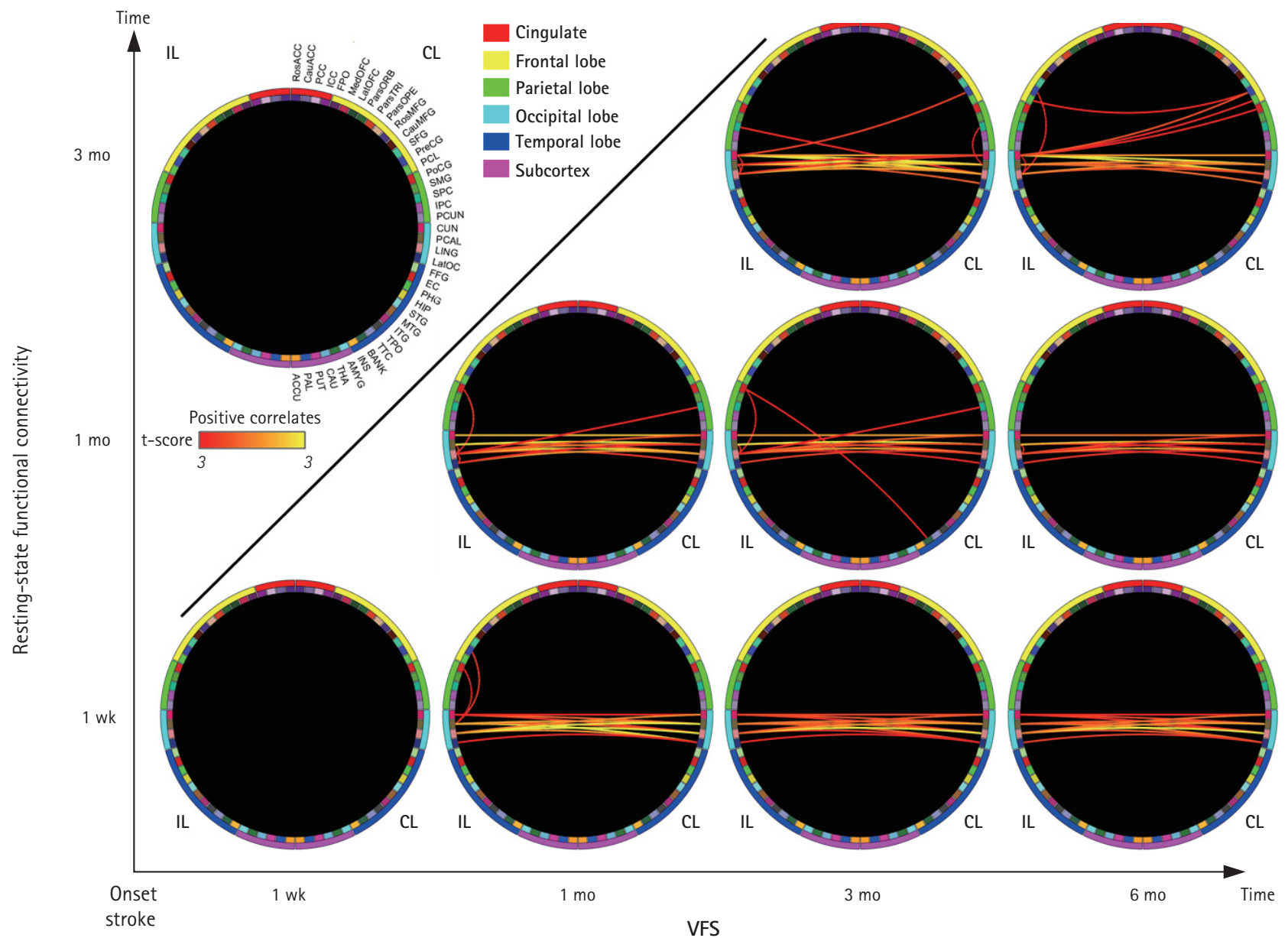

Figure 4. Correlation between resting-state functional connectivity (RSFC) and the visual field score (VFS; neurobehavioral correlates) over time. Significant levels of correlation analysis between RSFC and the VFS in the visual field defect patients $(n=32)$ are shown with red to yellow colors (yellow colors indicate higher positive correlation) in the Circos plot (row=time points of the RSFC; and column=time points of VFS). On the left-top of the figure, the region of interest (ROI) name abbreviations are shown (please see Supplementary Figure 2 for the full names of the ROIs). IL, ipsilesional hemisphere (i.e., left half of the Circos plot); $\mathrm{CL}$, contralesional hemisphere (i.e., right half of the Circos plot).

IL

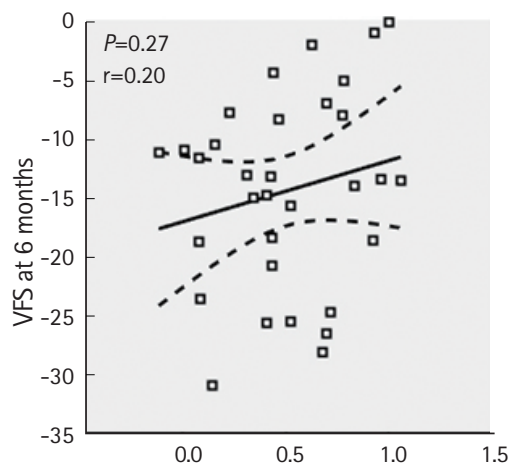

Average RSFC in occipital lobe within 1 week (Fisher z-score)
Between hemispheres

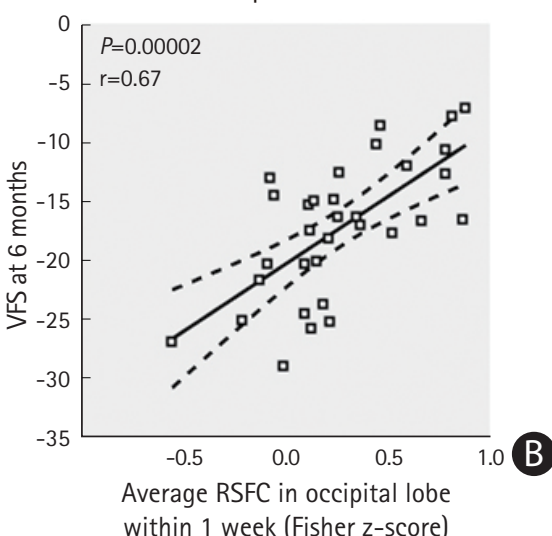

$\mathrm{CL}$

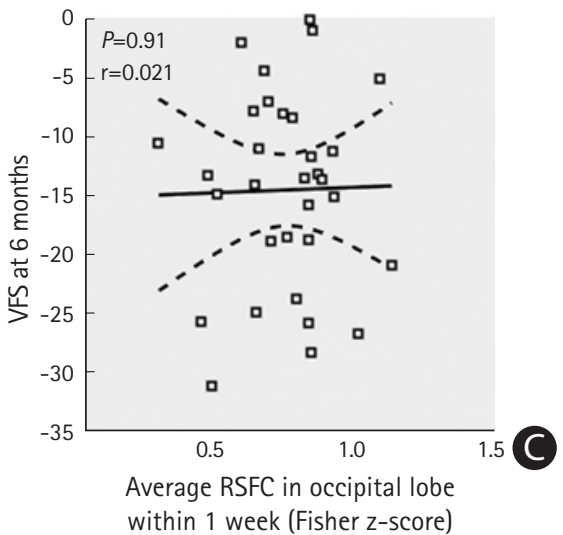

Figure 5. Correlation between early resting-state functional connectivity (RSFC) in the occipital lobe and visual field defect recovery. Scatter plots are shown between the average RSFC in the occipital lobe within 1 week and the visual field score (VFS) at 6 months after stroke. The regression line and its 95\% confidence intervals are shown by solid and dotted lines, respectively. (A) Average RSFC in IL occipital lobe regions of interest (ROI), (B) Average RSFC between hemispheres of occipital lobe ROI, and (C) Average RSFC in CL occipital lobe ROI. IL, ipsilesional hemisphere; CL, contralesional hemisphere. 
As an additional analysis of the visual cortex ROls including the cuneus, pericalcarine, lingual, and lateral occipital cortices, we calculated the average visual cortex RSFCs within the ipsiand contralesional hemispheres and between hemispheres, respectively (Figure 3). Compared to healthy controls, VFD patients showed lower levels of average visual cortex RSFC between the hemispheres (Bonferroni corrected $P=0.0001$, $P=0.044$, and $P=0.025$ at 1 week, 1 month, and 3 months after stroke onset, respectively). The average visual cortex RSFC between hemispheres was significantly increased over time (uncorrected $P=0.001, F[2,63]=7.6$ from repeated-measures ANO$V A$ across time; Bonferroni corrected $P=0.0027$ at 1 month and $P=0.013$ at 3 months from 1 week). The average visual cortex RSFC within the contralesional hemisphere was greater in patients than in controls (Bonferroni corrected $P=0.037$ at 1 week), then decreased at 3 months (uncorrected $P=0.04$ from 1 week). There were no significant differences between patients and healthy controls in the average visual cortex RSFC within the ipsilesional hemisphere (uncorrected $P=0.27$ at 1 week, $P=0.33$ at 1 month, and $P=0.32$ at 3 months).

\section{Alterations in neural behavior correlates after stroke}

Within 1 week after stroke, there were no significant neurobehavioral correlates between RSFC and the VFS. However, the interhemispheric RSFCs within the occipital lobe were positively correlated with the VFS at 1 month (NBS corrected $P=0.012$ ) and at 3 months (NBS corrected $P=0.009$ ) after stroke (Figure 4).

Interhemispheric RSFCs of the visual cortex at 1 week, 1 month, and 3 months were positively correlated with the later VFSs (NBS corrected $P=0.014, P=0.025$, and $P=0.021$ between 1 week RSFC and the 1-, 3-, and 6-month VFSs after stroke, respectively; $P=0.016$ and $P=0.046$ between the 1 -month RSFC and 3 - and 6-month VFS, respectively; and $P=0.011$ between the 3-month RSFC and 6-month VFS). Consistently, homotopic RSFCs of the cuneus, pericalcarine, and lingual cortices and heterotopic RSFCs between the pericalcarine and lingual cortices showed significant neurobehavioral correlates between the early interhemispheric RSFCs and later VFSs (uncorrected $P<0.0068, \mathrm{t}[30]>3.0$ for all RSFCs). Although the ipsilesional hemisphere RSFCs between the pericalcarine and motor-related ROIs (i.e., precentral and postcentral gyri) within 1 week showed positive correlations with VFSs at 1 month, the ipsilesional hemisphere RSFCs within 1 week were not significantly correlated with the 3- or 6-month VFSs.

The average interhemispheric RSFCs in the visual cortex ROIs including the cuneus, pericalcarine, lingual, and lateral occipital cortices within 1 week were positively correlated with the 6-month VFS. There were no significant neurobehavioral correlations between intrahemispheric RSFCs and the 6-month VFSs (Figure 5).

\section{Discussion}

In this study, we assessed the natural course of VFD recovery and RSFC in acute ischemic stroke patients with VFD. VFD recovery and neural changes occurred mostly within the first month, with an increase in the interhemispheric RSFCs in the visual cortex. In addition, positive neurobehavioral correlates were observed between the VFD status and interhemispheric RSFC in the visual cortex at 1 month. Most importantly, the initial and early interhemispheric RSFCs in the visual cortex could predict both the subacute and chronic stages of VFD prognosis.

With regard to the RSFC changes after stroke, interhemispheric RSFCs in the visual cortex (i.e., RSFCs including the cuneus, pericalcarine, and lingual cortex ROls) were found to be impaired after stroke in the unilateral visual cortex. The impaired interhemispheric RSFCs in the visual cortex recovered over time, although a lower level of RSFC was still observed at 3 months compared to healthy controls. Most of the significant increases in RSFC over time were observed within the first month after stroke onset, consistent with VFD recovery.

The early interhemispheric RSFCs in the visual cortices were correlated to good recovery of VFD. Previous studies showed that the interhemispheric RSFC was associated with spatial attention deficits and their recovery. ${ }^{26,27}$ Conversely, as in this study, the RSFC within a hemisphere, even within the damaged hemisphere, showed a weaker relationship with behavioral performance in other studies..$^{28,29}$ Our results suggest that brain interhemispheric connectivity is a key component for VFD status and clinical outcomes. Greater level of average visual cortex RSFC within the contralesional hemisphere was observed in patients than in controls at 1 week after stroke. The competition between hemispheres and the negative influence of activity in the intact hemisphere may be important to determine functional recovery. ${ }^{30,31}$ Recovery of these competitive interactions would have a beneficial effect in clinical recovery.

The critical period for VFD recovery appears to be within the first month, consistent with the RSFC change data in this study. Significant VFS improvements were observed during the recovery period. It is notable that visual dysfunction in the neurologically-unaffected hemifield was observed in this study (Figure 1A). Lower MTD in the unaffected hemifields compared with those of age-matched healthy controls were still observed up to 6 months after stroke onset. Moreover, the magnitude of improvement in the MTD in the affected hemifield was signifi- 
cantly greater than that in the unaffected hemifield. Previous studies have reported that visual-cortex lesions induce deficits in visual perception and processing speed in the intact hemifield. ${ }^{32,33}$ In vision-restoration studies, the authors proposed the "residual visual activation" theory, stipulating that repetitive stimulations (e.g., visual experience, visual training, or noninvasive electrical brain current stimulation) lead to the strengthening of synaptic transmission and synchronization of partially-damaged structures (within-system plasticity) and downstream neuronal networks (network plasticity). ${ }^{34}$ Disorganization and recovery of the interhemispheric RS functional network in the visual cortex is a plausible explanation for the dysfunction of the unaffected hemifield and VFD recovery of the affected hemifield, respectively.

In this study, several patients showed either negative or zero interhemispheric RSFCs in the visual cortex (the interhemispheric functional connectivity in Figure 5B). Several studies have shown that there is weaker activation and delayed temporal processing in the ipsilesional hemisphere than in the contralesional hemisphere. ${ }^{35-37} \mathrm{fMRI}$ shows brain activity by detecting changes associated with blood flow. The theoretical basis is that cerebral blood flow and neural activities are coupled. Insufficient blood supply in the ipsilesional hemisphere may explain the absence of functional connectivity between hemispheres in several patients. In addition, in the contralesional hemisphere, the intrahemispheric RSFC in the visual cortex decreased at 3 months compared to at 1 week, which may be attributable to decrease in compensatory increased blood flow in the contralesional hemisphere over time (Figure 3).

The interpretation of our findings regarding RSFC is based on the assumption that the correlations of MRI signals reflect those of neural signals. However, an important alternative explanation for the RSFC is that the correlation of MR signals reflect those of vascular oscillations, especially in very low frequency ranges. ${ }^{38-43}$ The decrease in interhemispheric RSFC, rather than in intrahemispheric RSFC, can be interpreted as originating from imbalance in blood supply between the hemispheres after stroke. ${ }^{44,45}$ Because vascular supply to only one hemisphere is affected after stroke, interhemispheric RSFC is expected to decrease to a greater extent than the RSFC within either the affected or intact hemisphere. In contrast, if the RSFC derives from neural signals, intrahemispheric RSFC would have been more greatly affected compared to the interhemispheric ones, since interhemispheric connections between the two primary visual cortices are confined to near the vertical meridian only, and those between higher visual areas are unlikely to be greater than the intrahemispheric connections, both feed-forward and feedback, among the visual areas.
Therefore, the positive correlation of RSFC and VF performance during recovery can be interpreted as arising from the restoration of the vascular supply to the lesioned hemisphere rather than from regeneration of neural connectivity. However, this cannot be sufficiently explained by the RSFC measures, as there is a lack of temporal landmarks to measure the disturbance of blood supply (e.g., task timing or contrast enhancement). Neurovascular and physiological mechanisms in RSFC measurements should be considered in the interpretation of our findings. ${ }^{4-47}$ Further task-based fMRI in combination with the measures of blood supply (e.g., using perfusion-weighted images) would be helpful to elucidate the neural recovery mechanism after stroke.

\section{Conclusions}

In conclusion, interhemispheric RSFCs in the visual cortex significantly increase over time after stroke. Functionally-reorganized RSFCs are positively correlated to the VFD status after stroke. The interhemispheric RSFC in the visual cortex within 1 week could be used as a biomarker to predict VFD recovery after stroke.

\section{Supplementary materials}

Supplementary materials related to this article can be found online at https://doi.org/10.5853/jos.2018.02999.

\section{Disclosure}

The authors have no financial conflicts of interest.

\section{Acknowledgments}

This study was supported by grants from the National Research Foundation of Korea (NRF) funded by the Korean government (MEST) (NRF-2014R1A2A1A11051280, NRF-2016R1C1B2015901 and NRF-2017R1A6A3A01001795), and the Korea Health Technology R\&D Project and Ministry for Healthcare and Welfare, Republic of Korea (HI12C1847 and HI18C2383).

\section{References}

1. Pollock A, Hazelton $C$, Henderson CA, Angilley J, Dhillon B, Langhorne $P$, et al. Interventions for visual field defects in patients with stroke. Cochrane Database Syst Rev 2011:CD008388.

2. Çelebisoy M, Çelebisoy N, Bayam E, Köse T. Recovery of visual-field defects after occipital lobe infarction: a perimetric 
study. J Neurol Neurosurg Psychiatry 2011;82:695-702.

3. Pambakian $A L$, Kennard C. Can visual function be restored in patients with homonymous hemianopia? Br J Ophthalmol 1997;81:324-328.

4. Zhang $X$, Kedar S, Lynn MJ, Newman NJ, Biousse V. Homonymous hemianopias: clinical-anatomic correlations in 904 cases. Neurology 2006;66:906-910.

5. Zhang X, Kedar S, Lynn MJ, Newman NJ, Biousse V. Natural history of homonymous hemianopia. Neurology 2006;66: 901-905.

6. Kim YH, You SH, Kwon YH, Hallett M, Kim JH, Jang SH. Longitudinal fMRI study for locomotor recovery in patients with stroke. Neurology 2006;67:330-333.

7. Puh U, Vovk A, Sevsek F, Suput D. Increased cognitive load during simple and complex motor tasks in acute stage after stroke. Int J Psychophysiol 2007;63:173-180.

8. Tombari D, Loubinoux I, Pariente J, Gerdelat A, Albucher JF, Tardy J, et al. A longitudinal fMRI study: in recovering and then in clinically stable sub-cortical stroke patients. Neuroimage 2004;23:827-839.

9. Ward NS. Functional reorganization of the cerebral motor system after stroke. Curr Opin Neurol 2004;17:725-730.

10. Biswal BB, Van Kylen J, Hyde JS. Simultaneous assessment of flow and BOLD signals in resting-state functional connectivity maps. NMR Biomed 1997;10:165-170.

11. Hampson M, Peterson BS, Skudlarski P, Gatenby JC, Gore JC. Detection of functional connectivity using temporal correlations in MR images. Hum Brain Mapp 2002;15:247-262.

12. Damoiseaux JS, Rombouts SA, Barkhof F, Scheltens $P$, Stam $\mathrm{CJ}$, Smith SM, et al. Consistent resting-state networks across healthy subjects. Proc Natl Acad Sci U S A 2006;103:1384813853.

13. Raichle ME. Cognitive neuroscience. Bold insights. Nature 2001;412:128-130.

14. Ogawa S, Lee TM, Kay AR, Tank DW. Brain magnetic resonance imaging with contrast dependent on blood oxygenation. Proc Natl Acad Sci U S A 1990;87:9868-9872.

15. Chen S, Ross TJ, Zhan W, Myers CS, Chuang KS, Heishman SJ, et al. Group independent component analysis reveals consistent resting-state networks across multiple sessions. Brain Res 2008;1239:141-151.

16. Lim JS, Kang DW. Stroke connectome and its implications for cognitive and behavioral sequela of stroke. J Stroke 2015;17: 256-267.

17. Minshew NJ, Keller TA. The nature of brain dysfunction in autism: functional brain imaging studies. Curr Opin Neurol 2010;23:124-130.

18. Zhang J, Meng L, Qin W, Liu N, Shi FD, Yu C. Structural dam- age and functional reorganization in ipsilesional $\mathrm{m} 1$ in wellrecovered patients with subcortical stroke. Stroke 2014;45: 788-793.

19. Jenkinson M, Beckmann CF, Behrens TE, Woolrich MW, Smith SM. FSL. Neuroimage 2012;62:782-790.

20. Van Dijk KR, Sabuncu MR, Buckner RL. The influence of head motion on intrinsic functional connectivity MRI. Neuroimage 2012;59:431-438.

21. Fischl B, Sereno MI, Tootell RB, Dale AM. High-resolution intersubject averaging and a coordinate system for the cortical surface. Hum Brain Mapp 1999;8:272-284.

22. Muschelli J, Nebel MB, Caffo BS, Barber AD, Pekar JJ, Mostofsky $\mathrm{SH}$. Reduction of motion-related artifacts in resting state fMRI using aCompCor. Neuroimage 2014;96:22-35.

23. Power JD, Mitra A, Laumann TO, Snyder AZ, Schlaggar BL, Petersen SE. Methods to detect, characterize, and remove motion artifact in resting state fMRI. Neuroimage 2014;84: 320-341.

24. Desikan RS, Ségonne F, Fischl B, Quinn BT, Dickerson BC, Blacker $\mathrm{D}$, et al. An automated labeling system for subdividing the human cerebral cortex on MRI scans into gyral based regions of interest. Neuroimage 2006;31:968-980.

25. Zalesky A, Fornito A, Bullmore ET. Network-based statistic: identifying differences in brain networks. Neuroimage 2010; 53:1197-1207.

26. Corbetta M, Kincade MJ, Lewis $C$, Snyder AZ, Sapir A. Neural basis and recovery of spatial attention deficits in spatial neglect. Nat Neurosci 2005;8:1603-1610.

27. He BJ, Snyder AZ, Vincent JL, Epstein A, Shulman GL, Corbetta M. Breakdown of functional connectivity in frontoparietal networks underlies behavioral deficits in spatial neglect. Neuron 2007;53:905-918.

28. Carter AR, Astafiev SV, Lang CE, Connor LT, Rengachary J, Strube MJ, et al. Resting interhemispheric functional magnetic resonance imaging connectivity predicts performance after stroke. Ann Neurol 2010;67:365-375.

29. Dilks DD, Serences JT, Rosenau BJ, Yantis S, McCloskey M. Human adult cortical reorganization and consequent visual distortion. J Neurosci 2007;27:9585-9594.

30. Murase N, Duque J, Mazzocchio R, Cohen LG. Influence of interhemispheric interactions on motor function in chronic stroke. Ann Neurol 2004;55:400-409.

31. Heiss WD, Kessler J, Thiel A, Ghaemi M, Karbe H. Differential capacity of left and right hemispheric areas for compensation of poststroke aphasia. Ann Neurol 1999;45:430-438.

32. Bola M, Gall C, Sabel BA. The second face of blindness: processing speed deficits in the intact visual field after pre- and post-chiasmatic lesions. PLoS One 2013;8:e63700. 
33. Bola M, Gall C, Sabel BA. "Sightblind": perceptual deficits in the "intact" visual field. Front Neurol 2013;4:80.

34. Sabel BA, Henrich-Noack P, Fedorov A, Gall C. Vision restoration after brain and retina damage: the "residual vision activation theory". Prog Brain Res 2011;192:199-262.

35. Goebel R, Muckli L, Zanella FE, Singer W, Stoerig P. Sustained extrastriate cortical activation without visual awareness revealed by fMRI studies of hemianopic patients. Vision Res 2001;41:1459-1474.

36. Nelles G, Widman G, de Greiff A, Meistrowitz A, Dimitrova A, Weber $J$, et al. Brain representation of hemifield stimulation in poststroke visual field defects. Stroke 2002;33:1286-1293.

37. Schoenfeld MA, Noesselt T, Poggel D, Tempelmann C, Hopf $J M$, Woldorff MG, et al. Analysis of pathways mediating preserved vision after striate cortex lesions. Ann Neurol 2002; 52:814-824.

38. Power JD, Cohen AL, Nelson SM, Wig GS, Barnes KA, Church $J A$, et al. Functional network organization of the human brain. Neuron 2011;72:665-678.

39. Yeo BT, Krienen FM, Sepulcre J, Sabuncu MR, Lashkari D, Hollinshead $M$, et al. The organization of the human cerebral cortex estimated by intrinsic functional connectivity. J Neurophysiol 2011;106:1125-1165.

40. Doucet G, Naveau M, Petit L, Delcroix N, Zago L, Crivello F, et al. Brain activity at rest: a multiscale hierarchical functional organization. J Neurophysiol 2011;105:2753-2763.

41. Hacker CD, Laumann TO, Szrama NP, Baldassarre A, Snyder $A Z$, Leuthardt $E C$, et al. Resting state network estimation in individual subjects. Neuroimage 2013;82:616-633.

42. Cole MW, Bassett DS, Power JD, Braver TS, Petersen SE. Intrinsic and task-evoked network architectures of the human brain. Neuron 2014;83:238-251.

43. Gordon EM, Laumann TO, Adeyemo B, Huckins JF, Kelley WM, Petersen SE. Generation and evaluation of a cortical area parcellation from resting-state correlations. Cereb Cortex 2016; 26:288-303.

44. Siegel JS, Snyder AZ, Ramsey L, Shulman GL, Corbetta M. The effects of hemodynamic lag on functional connectivity and behavior after stroke. J Cereb Blood Flow Metab 2016;36: 2162-2176.

45. Siegel JS, Shulman GL, Corbetta M. Measuring functional connectivity in stroke: approaches and considerations. $J$ Cereb Blood Flow Metab 2017;37:2665-2678.

46. Mandeville ET, Ayata C, Zheng Y, Mandeville JB. Translational MR neuroimaging of stroke and recovery. Trans/ Stroke Res 2017;8:22-32.

47. Veldsman M, Cumming T, Brodtmann A. Beyond BOLD: optimizing functional imaging in stroke populations. Hum Brain Mapp 2015;36:1620-1636. 